\title{
Tricking the System / Tricked by the System
}

By N.N.

- Desideratum

Evernote keywords: issues tagged for first-priority treatment in this entry

- fraud

- scandal (after the tests were diffused online)

- school exams

- school teacher $v s$. students

Entries pointing here

ARRAYS - Baby Milk $\downarrow$ Commemoration $/$ Memorial Days $\diamond$ Conversions $\diamond$ Crowdfunding $\diamond$ Father Figures

CODES - True vs. False

CODES COLLAPSED - True $=$ False $($ Life in Limbo $)$

4...@... 